\title{
BMJ Open GETUG-AFU 31: a phase I/II multicentre study evaluating the safety and efficacy of salvage stereotactic radiation in patients with intraprostatic tumour recurrence after external radiation therapy - study protocol
}

David Pasquier, ${ }^{\oplus, 2}$ Marie-Cécile Le Deley, ${ }^{3}$ Emmanuelle Tresch, ${ }^{3}$ Luc Cormier, ${ }^{4}$ Martine Duterque, ${ }^{5}$ Soazig Nenan, ${ }^{6}$ Eric Lartigau ${ }^{1,2}$

To cite: Pasquier D, Le Deley M-C, Tresch E, et al. GETUG-AFU 31: a phase $1 / \mathrm{ll}$ multicentre study evaluating the safety and efficacy of salvage stereotactic radiation in patients with intraprostatic tumour recurrence after external radiation therapystudy protocol. BMJ Open 2019;9:e026666. doi:10.1136/ bmjopen-2018-026666

- Prepublication history and additional material for this paper are available online. To view these files, please visit the journal online (http://dx.doi. org/10.1136/bmjopen-2018026666).

Received 13 September 2018 Revised 10 July 2019 Accepted 15 July 2019

A) Check for updates

(C) Author(s) (or their employer(s)) 2019. Re-use permitted under CC BY-NC. No commercial re-use. See rights and permissions. Published by BMJ.

For numbered affiliations see end of article.

Correspondence to

Dr David Pasquier;

d-pasquier@0-lambret.fr

\section{ABSTRACT}

Introduction Prostate cancer is the third most important cancer in terms of mortality in men. No standard local treatment exists for patients with an intraprostatic recurrence after radiotherapy. Stereotatic body radiotherapy (SBRT) could be a curative treatment for local recurrence. The phase $\mathrm{l} / \mathrm{ll}$ primary objective is the selection of the recommended dose for salvage-SBRT and to estimate the efficacy.

Methods and analysis We plan to perform a multicentre prospective phase $\mathrm{I} / \mathrm{Il}$ study including at least 47 patients. Eligible patients are patients with biochemical recurrence occurring at least 2 years after external radiotherapy for prostatic adenocarcinoma by the Phoenix definition (prostate-specific antigen (PSA) nadir $+2 \mathrm{ng} / \mathrm{mL}$ ) and histologically proven intraprostatic recurrence only (stage T1-T2 on relapse, PSA level $\leq 10 \mathrm{ng} / \mathrm{mL}$, PSA doubling time $>10$ months, absence of pelvic or metastatic recurrence proven by choline or PSMA positron emission tomography scan, and pelvic and prostatic assessment by multiparametric MRI). The phase I primary objective is the selection of the recommended dose for salvage-SBRT $(5 \times 6,6 \times 6$ or $5 \times 5$ Gy) based on dose-limiting toxicity (DLT). The dose of salvage-SBRT will be selected using a time-to-event continual reassessment method based on DLT defined as grade $\geq 3$ gastrointestinal or urinary toxicity or any other grade 4 adverse event. The phase II primary outcome is to estimate the efficacy of the salvageSBRT in terms of biochemical relapse-free survival rate (Phoenix definition: increase in serum total PSA $\geq 2 \mathrm{ng} / \mathrm{mL}$ above the nadir). Phase II secondary outcomes are acute and late toxicities, quality of life, clinical progression-free survival defined as the time interval between the date of registration and the date of clinical progression or death irrespective of the cause.

Ethics and dissemination The study has received ethical approval from the Ethics committee 'Ile-de-France III'. Academic dissemination will occur through publication and conference presentations.

Trial registration number NCT03438552
Strengths and limitations of this study

- Very innovative trial evaluating stereotactic body radiotherapy for recurrent prostate cancer, the only ongoing trial of this kind in Europe to our knowledge.

- Clinical trial supported by the GETUG-AFU cooperative group, expert in the field.

- Time-to-event continual reassessment method (TITE-CRM), a more appropriate method than the $3+3$ design to quantify late toxicity in phase I radiotherapy trials.

- Proof-of-concept study; further research will be required.

Small sample size

\section{BACKGROUND}

Prostate cancer is the third most important cancer in terms of mortality in men (after lung and colorectal cancer), and the fourth leading cancer overall. ${ }^{1}$ In the European Union, it was predicted that 72600 patients would die from prostate cancer in 2015 . The rate of recurrence/relapse of prostate cancer after primary external beam radiotherapy varies from $21 \%$ to $65 \%$ depending on the study. ${ }^{23}$ Zelefsky et al performed biopsies on 339 patients after treatment with 3-dimensional conformal radiotherapy or intensity modulated RT for T1c-T3 stage prostate cancer, with a minimum follow-up of 2.5 years. ${ }^{2}$ They found that the rate of positive biopsy according to radiation dose was $65 \%$ for $<70.2 \mathrm{~Gy}, 38 \%$ for $70.2 \mathrm{~Gy}, 27 \%$ for $75.6 \mathrm{~Gy}$ and $25 \%$ for $\geq 81 \mathrm{~Gy}$. A 2-year biopsy was performed in 312/843 patients included in MRC RT01 trial. A positive biopsy was prognostic of worse biological progression-free survival (bRFS) compared with negative 
and suspicious biopsies, HR=4.81 (95\% CI 2.50 to 9.26, $\mathrm{p}<0.001)$. The estimate for survival was $\mathrm{HR}=1.58$ (95\% CI 0.52 to $4.78, \mathrm{p}=0.42$ ).

In the literature and guidelines, a minimum time of 2 years is recommended between radiotherapy and prostate biopsies to minimise the risk of false positive and this 2-year interval has been selected in our study. This risk decreases over time. Nevertheless, the guidelines recommend to perform prostate biopsies before any salvage treatment, and the diagnosis is based on imaging too in our study.

D'Amico et al reported that the 5-year prostate cancer mortality rate after biochemical recurrence in patients who received external beam radiotherapy for localised prostate cancer depended on the pretreatment Gleason score: $24 \%$ for $\leq 6$ score, $40 \%$ for $3+4$ score and $59 \%$ for $4+3$ or higher score $(\mathrm{p}=0.01)$; as well as, on the pretreatment prostate-specific antigen (PSA) level: 22\% for $\leq 10 \mathrm{ng} / \mathrm{mL}, 40 \%$ for $>10$ and $\leq 20 \mathrm{ng} / \mathrm{mL}$ and $60 \%$ for $>20 \mathrm{ng} / \mathrm{mL}(\mathrm{p}=0.04) .{ }^{4} \mathrm{In}$ addition, Buyyounouski et al reported that the interval to biochemical recurrence was an important factor in identifying men at high risk of distant metastasis and death. ${ }^{5}$ An interval to biochemical recurrence (PSA nadir $+2 \mathrm{ng} / \mathrm{mL}$ ) of $<18$ versus $\geq 18$ months was associated with a 5 -year distant metastasis rate of $52 \%$ versus $20 \%(\mathrm{p}<0.0001)$, and a prostate-cancer specific mortality rate of $36 \%$ versus $6 \% \quad(p=0.0001)$. Thus, late relapse and long PSA doubling time are prognostic factors of solely intraprostatic relapse.

PSA is a validated biomarker for prostate cancer. PSA levels are extensively used to monitor response to radical prostatectomy (RP) and external-beam radiotherapy (EBRT). The serum PSA level is an important surrogate for recurrence in prostate cancer patients. The recommendations of the Radiation Therapy Oncology Group (RTOG) ASTRO Phoenix Consensus Conference suggested that an increase of $\geq 2 \mathrm{ng} / \mathrm{mL}$ from the nadir be used to define recurrence/relapse. ${ }^{6}$ It is important to note that biochemical recurrence may indicate local or systemic disease recurrence, and may require prostate biopsy for confirmation particularly when local salvage treatments are being considered. ${ }^{7}$

A number of different salvage treatments have been used after failure of primary radiotherapy. RP is more invasive than brachytherapy, ultrasound, high-intensity focused ultrasound (HIFU) and stereotatic body radiotherapy (SBRT). Below is a brief discussion of the results obtained with each techniques and its associated toxicity and complications.

Salvage RP (SRP) is a treatment option after local recurrence following EBRT. However, the morbidity, including incontinence and erectile dysfunction, is higher than that observed with first-line RP patients. A systematic literature review $^{7}$ reported that the probability of bRFS following SRP in prostate cancer patients was $47 \%-82 \%$ after 5 years and $28 \%-53 \%$ after 10 years. SRP has an increased risk of adverse events due to fibrosis and poor wound healing after radiotherapy. Furthermore, SRP after radiotherapy compared with primary RP has a significantly higher rate of urinary and gastrointestinal morbidity. ${ }^{8}$ The review above $^{7}$ reported that the most frequent complications were anastomotic stricture $(7 \%-41 \%)$ and rectal injury $(0 \%-28 \%)$. The majority $(50 \%-91 \%)$ of men had erectile dysfunction before SRP and $80 \%-100 \%$ after surgery. Postoperative urinary continence ranged from $21 \%$ to $90 \%$.

In a recent review, salvage brachytherapy after EBRT is reported to achieve biochemical control rates of $20 \%-$ 89\% (median follow-up: 19-108 months). ${ }^{9}$ Rates of genitourinary toxicities range from $12 \%$ to $87 \%$ for grade $1-2$, and $3 \%$ to $47 \%$ for grade $3-4$ toxicities. Similarly, the rates of gastrointestinal toxicities range from $4 \%$ to $65 \%$ for grade $1-2$, and $0 \%$ to $20 \%$ for grade $3-4$ toxicities. Erectile dysfunction was observed in $2 \%-95 \%$ of men. A phase II study of salvage high-dose-rate brachytherapy (HDR) for treating recurrent prostate cancer after definitive external beam radiotherapy was reported by Yamada et al. ${ }^{10}$ The 42 patients enrolled, with biopsy proven recurrence, were treated with salvage HDR (iridium-192) with a resulting bRFS at 5 years of $68.5 \%$ (median follow-up of 36 months (range: 2-66 months)). Acute genitourinary toxicity was observed: grade $1,38 \%$ of patients and grade 2: $40 \%$. Similarly, late genitourinary toxicity was observed: grade $1,38 \%$ of patients and grade $2,48 \%$. In addition, one patient had a grade 3 urinary incontinence and three had grade 3 urethral strictures. Late gastrointestinal toxicities were observed: grade 1, $43 \%$ of patients and grade 2 , $14 \%$. More recently, a study of 83 prostate cancer patients with local recurrence after radiotherapy treated with HDR was reported. ${ }^{11}$ The 3-year and 5-year bRFS were $76 \%$ and $67 \%$. A phase II study of the RTOG in 100 patients was recently presented: $12(14 \%)$ experienced late grade 3 genitourinary/gastrointestinal adverse effects. This rate of late grade 3 toxicity (14\%) was not unacceptable by the predetermined protocol specification, without any grade 4-5 events. The only factor predictive of late toxicity was implant dose, underlining the need for meticulous planning and technique to limit the final delivered dose. ${ }^{12}$ In France, a phase II study, 'Brachytherapy for Recurrent Prostate Cancer' (CAPRICUR) was recently completed. ${ }^{13}$

HIFU is another less invasive salvage treatments following recurrence. HIFU uses focused ultrasound to generate heat $\left(85^{\circ} \mathrm{C}\right)$ which induces tissue necrosis. ${ }^{14}$ The following studies have investigated the use of salvageHIFU after initial radiotherapy. A French group treated 290 men with biopsy-confirmed recurrent prostate cancer, after radiotherapy, with salvage HIFU. ${ }^{15}$ At 7 years, the cancer-specific survival rate was $80 \%$ and the metastasis-free survival rate was $79.6 \%$. Rectourethral fistula occurred in $0.4 \%$ of patients and $19.5 \%$ had grade $2 / 3$ incontinence. Half of the patients also received hormone therapy. Survival without relapse at 5 years after HIFU, by D'Amico risk groups prior to their initial treatment, was $45 \%$ (favourable), $31 \%$ (intermediate risk) and $21 \%$ (high risk). In this cohort, the grade $\leq 3$ urinary incontinence levels were 23\% (favourable), $14 \%$ (intermediate 
risk) and $9 \%$ (high risk). Nearly $8 \%$ of patients required an artificial sphincter following HIFU. Importantly, pubic osteitis occurred in $2.5 \%$ of patients despite adherence to parameters specific to HIFU following radiotherapy. ${ }^{15}$

Cryotherapy is thermoablative treatment; the third-generation argon/helium-based cryotherapy system creates precise isotherms through ultrathin needles. ${ }^{16}$ In a retrospective multicentre series pooling 279 patients, survival without biochemical relapse at 5 years was $54 \%$. Prostatic biopsies showed tumour persistence in $32 \%$ of patients following cryotherapy. ${ }^{17}$ In a paired case-controlled study, prostatectomy and cryotherapy were compared following radiotherapy. Survival without relapse (PSA nadir $+0.4 \mathrm{ng} / \mathrm{mL}$ ) at 5 years was significantly lower after cryotherapy (21\% vs $61 \%, \mathrm{p}<0.001)$; this was confirmed by the 5-year OS rate of $85 \%$, cryotherapy, versus $95 \%$, RP $(p=0.001){ }^{18}$ Intermediate results from a study investigating third-generation cryotherapy as a salvage treatment of locally recurrent prostate cancer after radiotherapy was published. ${ }^{19}$ The 5-year bRFS rate (Phoenix definition) was $43.5 \%$ and with a 5 -year OS rate of $92.3 \%$. Mild complications (grades 1 and 2) were reported including: mild incontinence $(9.4 \%)$, acute rectal pain $(31.3 \%)$, lower urinary tract symptoms $(15.6 \%)$ and erectile dysfunction $(57.1 \%)$. Grade 3 toxicities: incontinence $(3.1 \%)$ and urethral sloughing $(3.1 \%)$ were observed.

Androgen-deprivation therapy (ADT) alone (continuous or intermittent) is commonly given to patients with biochemical relapse after radiotherapy. ${ }^{20}$ In a series based on data from a North American national registry (CaPSURE), $71 \%$ of patients were treated for biochemical relapse after prostatectomy or radiotherapy. ADT was initiated in $93 \%$ of these patients, and the remaining patients were treated with surgery, brachytherapy, cryotherapy or repeat external radiotherapy. ${ }^{21}$ ADT may cause adverse effects impacting patient's quality of life (including hot flushes, erectile dysfunction and reduced libido, cognitive impairment and anaemia). The metabolic changes associated with hormone therapy may also increase the risk of cardiovascular morbidity. The reduction in bone mass is maximal in the first year and increases with the duration of castration; the risk of fracture is increased in patients surviving for $>5$ years. ${ }^{22}{ }^{23}$ The guidelines suggest that a simple follow-up can be implemented for local recurrence in patients with a limited life expectancy or for those who do not wish to undergo local salvage treatment. The last European Association of Urology guidelines ${ }^{24}$ recommended to perform salvage surgery in experienced centres due to the increased rate of side effects. It is recommended to offer/discuss HIFU, cryosurgical ablation and salvage brachytherapy to/with patients without evidence of metastasis and with histologically proven local recurrence and to inform patients about the experimental nature of these approaches. The level of evidence for each of these recommendations is $3 .^{24}$

SBRT delivers highly conformal, high-dose radiation in a few fractions (hypofractionation), typically $5-7$ fractions for prostate cancer. It is reported that tissues with a low $\alpha / \beta$ ratio, as for prostate cancer, are more sensitive to large doses of radiation per fraction. The surrounding normal tissue could have similar or higher $\alpha / \beta$ ratio. This suggests that hypofractionation (large radiation dose per fraction) may result in improved tumour control with limited toxicity. A pooled analysis of 1100 patients included in separate prospective phase II studies in eight institutions was performed to evaluate the effectiveness of SBRT as a first-line treatment for localised prostate cancer. ${ }^{25}$ The SBRT was delivered by CyberKnife with a median dose of $36.25 \mathrm{~Gy}$ in 4-5 fractions. The 5-year bRFS rate was $93 \%$ for all patients. Thus, relapse-free survival rates after SBRT compare favourably with other definitive treatments for low and intermediate-risk patients with a short follow-up. SBRT is well tolerated with a low effect on quality of life. ${ }^{25-28}$ In addition, sexual function appeared to be spared in the majority of patients. ${ }^{25-28}$

SBRT has also been used as a salvage treatment following failure of external radiotherapy. Jereczek-Fossa et al published data on 34 consecutive patients treated with robotic SBRT for isolated recurrent primary, lymph node or metastatic prostate cancer. ${ }^{29}$ Of the 34 patients, 15 patients had intraprostatic recurrence, confirmed by biopsy and were treated with SBRT (CyberKnife) with a median dose of $30 \mathrm{~Gy}$ in 5 fractions. Fourteen patients showed a biochemical response; the median survival without recurrence was 13 months. Five patients presented a clinical relapse, including one new intraprostatic recurrence. Urinary toxicity was low, with a grade 3 urinary toxicity in only one patient. No case of acute or late gastrointestinal toxicity was reported. This good gastrointestinal tolerability has subsequently been confirmed. ${ }^{29-32}$ In Fuller et al, 29 patients were treated in a phase II trial with stereotactic radiotherapy for intraprostatic recurrence. ${ }^{30}$ Eligible patients had to present biopsy-proven intraprostatic recurrence graded T1 to T3N0M0 $>2$ years postradiotherapy and without a pre-existing grade $>1$ radiotherapy toxicity. The prior median dose delivered was $73.8 \mathrm{~Gy}$ and the median interval between the treatments was 88 months. The dose delivered to the entire gland was $34 \mathrm{~Gy}$ in 5 fractions over 5 days (heterogeneous intraprostatic dose of up to $50 \mathrm{~Gy}$ ). With a median follow-up of 24 months, survival without recurrence was $82 \%$. Toxicity was acceptable, with $18 \%$ grade $\geq 2$ urinary toxicity, including one patient with a grade 4 toxicity, and no late gastrointestinal toxicity above grade 1 . Particular attention should be given to patients who still exhibit urinary toxicity after initial radiotherapy. ${ }^{30}$ Our preliminary retrospective results in 23 patients treated for this indication were published recently. ${ }^{31} \mathrm{~A}$ total dose of $36 \mathrm{~Gy}$ was prescribed in 6 fractions of $6 \mathrm{~Gy}$. Nineteen patients had a whole-gland and four a partial treatment. Median follow-up was 23 months (range 6-40 months). We observed no grade 4 or 5 toxicity. Two patients presented grade 3 toxicities. Others toxicities include urinary (5 grade 2 and 9 grade 1 ) and rectal toxicities (2 grade 2 and 2 grade 1). The dose levels selected in our study are 
close to those used in de novo patients, and the same as those described in retrospective salvage treatment series. These schemes seem to provide an acceptable compromise between efficacy and toxicity but have not been evaluated prospectively.

To date, no standard local treatment exists for patients with an intraprostatic recurrence after radiotherapy. ${ }^{33}$ A number of treatments options exist including RP, brachytherapy, HIFU, cryotherapy and stereotactic radiotherapy. The literature to date consists mainly of retrospective and small prospective series making it difficult to assess and compare these techniques. In recent years, SBRT has been used to treat localised prostate cancer in the primary setting but also as a salvage treatment after failure of radiotherapy. The initial results of these retrospective studies are promising, with respect to survival and tolerance, but further studies are required to confirm these initial results. Our proposed study will provide further evidence of SBRT as a supplementary non-invasive curative treatment for local recurrence following radiotherapy. This study could provide the foundation for prospective studies comparing the available salvage treatments after radiotherapy.

\section{METHODS/DESIGN}

This study is approved by the Ethics committee 'Ile de France III' (2017-A00008-45) and is registered on clinicaltrials.gov. This multicentre open-labelled phase I/II study will initially select the SBRT scheme (phase I), either $5 \times 6$, $6 \times 6$ or $5 \times 5$ Gy. The effectiveness of SBRT scheme selected in phase I will then be evaluated in a single-arm multicentre phase II study.

\section{Phase I: primary objective and assessment}

Selection of the recommended dose for salvage-SBRT (either $5 \times 6,6 \times 6$ or $5 \times 5 \mathrm{~Gy}$ ) based on dose-limiting toxicity (DLT) observed during the 18 weeks following the initiation of salvage-SBRT. The dose of salvage-SBRT will be selected using a TITE-CRM ${ }^{34-36}$ based on DLT defined as grade $\geq 3$ gastrointestinal or urinary toxicity or any other grade 4 adverse event observed during the 18 weeks following the initiation of salvage-SBRT.

\section{Phase II: primary objective and assessment}

Estimate the efficacy of the salvage-SBRT in terms of bRFS rate (Phoenix definition: increase in serum total $\mathrm{PSA} \geq 2 \mathrm{ng} / \mathrm{mL}$ above the nadir). Time to bRFS will be computed from registration. Patients alive without biochemical progression at the time of the analysis will be censored at the last follow-up date. In the event of death, whatever the cause of death, the patient will be considered as a failure.

\section{Phase II: secondary objective(s) and assessment}

1. Acute and late genitourinary and gastrointestinal toxicities over the first 3 years according to the NCI-CTCAE V.4.03 classification (14 June 2010), International
Prostate Symptom Score (IPSS) score for urinary symptoms and International Index of Erectile Function for erectile function. Patients will be followed for 5 years after salvage SBRT to assess late toxicity. Patients with second biochemical recurrence will not be excluded in order to assess late toxicity.

2. Quality of life will be evaluated based on the European Organization for Research and Treatment of Cancer (EORTC) Quality of Life Questionnaire (QLQ)-C30 and QLQ-prostate specific 25-item (PR25) scales. The time until definitive deterioration will be computed from registration until the first observation of a definitive deterioration of the quality of life, defined as a score decreased by 10 points (in the case of global health scale and functional scales) or increased by 10 points (in the case of symptom scales) compared with the score at baseline, without later improvement superior to 10 points compared with baseline score.

3. Clinical progression-free survival is defined as the time interval between the date of registration and the date of clinical progression (local progression assessed by the physical examination, or appearance of metastatic lesions) or death irrespective of the cause.

4. Overall survival is defined as the time interval between the date of registration and the date of death irrespective of the cause.

5. Erythroblast transformation-specific related gene (ERG) fusion expression will be evaluated using immunohistochemistry tests. ERG fusion and androgen-receptor splice variant 7 expression will be evaluated on biopsies before the first radiotherapy treatment (at diagnosis, if available) and before salvage radiotherapy after biochemical recurrence.

\section{Diagnosis and inclusion criteria}

1. Biochemical recurrence occurring at least 2 years after external radiotherapy for prostatic adenocarcinoma by the Phoenix definition (PSA nadir $+2 \mathrm{ng} / \mathrm{mL}$ )

2. T1-T2c and PSA $\leq 20 \mathrm{ng} / \mathrm{mL}$ and Gleason score $\leq 7$ at initial diagnosis of prostate cancer before the initial/ first treatment.

3. Recurrence of prostatic adenocarcinoma proven by histology following radiotherapy by transrectal or transperineal sextant biopsies of the two lobes of the prostate, with a minimum of 12 biopsies, irrespective of Gleason score. Biopsies of the seminal vesicles are optional.

4. Clinical stage T1-T2 on relapse; unilateral extracapsular extension (T3a) on MRI permitted except posteriorly relative to the rectum

5. Estimated clinical target volume (CTV)/prostate volume $<0.5$ based on imaging and biopsies.

6. Pelvic and prostatic assessment by multiparametric (mp) MRI.

7. Absence of pelvic or metastatic recurrence proven by choline positron emission tomography (PET) scan.

8. Performance status WHO $0-1$. 
9. PSA level $\leq 10 \mathrm{ng} / \mathrm{mL}$ at baseline (before salvage-SBRT).

10. PSA doubling time $>10$ months.

11. IPSS $\leq 12$.

12. Uroflowmetry with a maximum flow rate $>10 \mathrm{~mL} / \mathrm{s}$, a postvoid residual urine volume $<150 \mathrm{~mL}$ and a urine volume $>150 \mathrm{~mL}$.

13. No other anticancer treatment since the external radiotherapy administered as first-line treatment.

14. No other anticancer treatment planned for the current recurrence.

15. No contraindication to fiducial marker implants; haemostatic disorders must be corrected before implantation.

16. Age $>18$ years.

17. Life-expectancy $\geq 5$ years (Lee scale).

18. Patient registered with a health insurance system.

19. Patient who has signed the informed consent form.

20. Patients willing and able to comply with the scheduled visits, treatment plan, laboratory tests and other study procedures indicated in the protocol.

\section{Exclusion criteria}

1. Lymph node or metastatic spread.

2. Late postradiotherapy urinary or gastrointestinal toxicity of grade $\geq 2$ (following primary radiotherapy).
3. Other cancers in the last 5 years except for non-melanoma-type skin cancer.

4. History of inflammatory bowel disease.

5. Anticoagulant treatment.

6. Contraindications to undergoing MRI.

7. Prostate volume $>80 \mathrm{cc}$.

8. Transurethral resection of the prostate in the 6 months before registration.

9. Presence of rectal telangiectasia grade 3 classified by the Vienne Proctoscopy Score (obligatory proctoscopy). ${ }^{3738}$

10. Previous rectal surgery

11. Patients unable to undergo medical follow-up in the study for geographical, social or psychological.

12. Person deprived of their liberty or under protective.

\section{Intervention}

A flowchart presenting the different steps from inclusion until treatment is presented in figure 1. Five or six fractions, at a level of 5 or 6 Gy per session (either $5 \times 6,6 \times 6$ or $5 \times 5 \mathrm{~Gy}$ ), will be delivered over a maximum of 12 days to provide a total dose of 25-36 Gy. This radiotherapy may be administered with the CyberKnife or a linear accelerator allowing stereotactic radiotherapy. The patient will be placed in a stable and reproducible supine position. Intraprostatic markers (fiducials) will be implanted

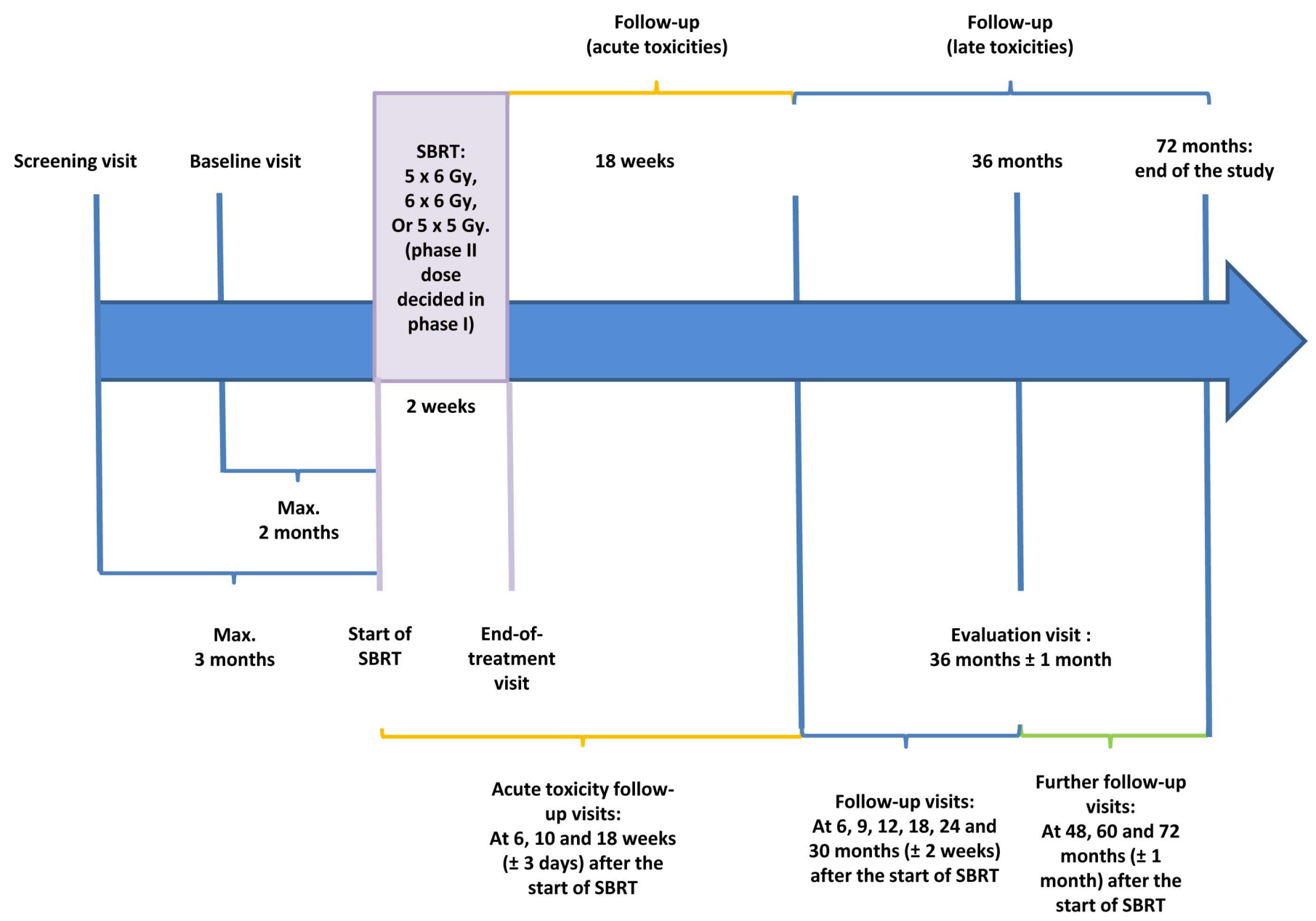

Figure 1 Overview of study flow chart. SBRT, stereotactic body radiotherapy. 
before the dosimetric CT scan and MRI are performed. The choice of markers is left to the discretion of each participating site (gold seeds and so on). The site needs to ensure that the repositioning of the prostate is precise $(\leq 2 \mathrm{~mm})$, allowing an exact overlay between dosimetric MRI and CT scan. The implant of fiducials is obligatory irrespective of the stereotactic radiotherapy technique used. The fiducials can be implanted during or after the biopsies. The CT scans and MRIs registration is guided by the fiducials. Each team can choose the fiducials visible on the MRI they wish to use and/or integrate a specific sequence during the centering MRI for better visualisation.

An mpMRI and CT scan will be carried out at least 1 week after fiducial implantation. CT scan images should be acquired with the patient in the treatment position using the chosen immobilising system, if required according to centres' standard procedures. An intravenous injection of a contrast product should be administered unless contraindicated. Acquisition should allow anatomical structures and markers (already implanted in the prostate) to be visualised. The bladder will be halffilled (neither full nor empty, ie, a final voiding $90 \mathrm{~min}$ before the CT scan followed by ingestion of $250-350 \mathrm{~mL}$ of water). The rectum will be empty and not distended. If necessary rectal enema can be used before CT scan acquisition. Contiguous CT scan slices $\leq 2 \mathrm{~mm}$ thick will be taken between the L5-S1 joint space and the small trochanters. After image transfer to a planimetric console, fiducial-based registration with the prostatic mpMRI will take place in order to provide a better definition of the gross target volume (GTV) and prostatic contours, especially the apex. Fiducial-based CT-MRI registration is mandatory. Multimodality image registration with choline-PET is possible but not mandatory.

Delineation of the target volume will be carried out by a radiotherapist experienced in the definition of prostate volumes on CT scans and MRIs. The mpMRI-defined contour will be integrated with the CT scan derived contours in order to define tumour and the prostatic apex more precisely. GTV will be represented by lesion defined on the multiparametric MRI \pm choline-PET; a 5-7 mm margin around the GTV will be used to define the CTV. CTV is contained in the prostate, except in the case of extracapsular extension (unilateral extracapsular extension on MRI permitted except posteriorly relative to the rectum). In the case where the positive biopsy(ies) are outside and adjacent to the visible lesions on the MRI, the zone containing these biopsies must be included in the CTV so that the prostate cancer recurrence not visible on the MRI is included in the CTV. If no lesion is visible on the MRI and/or choline-PET, the zone containing positive biopsies must be included in the CTV. For example: MRI \pm choline-PET lesion in $3 p$ and $4 p$ according to ESUR guidelines, with positive biopsies in $1 \mathrm{p}, 3 \mathrm{p}$ and $4 \mathrm{p}$ : CTV=MRI GTV $+5 \mathrm{~mm}+$ the posterior right base. The total CTV should not be more than half of the total volume of the prostate by MRI. The planning target

\begin{tabular}{lll}
\hline \multicolumn{2}{l}{ Table 1 Organs at risk constraints } \\
\hline Rectum wall & Bladder wall & Urethra $+\mathbf{3} \mathbf{m m}$ \\
\hline V27 Gy $<2$ cc & V27 Gy $<5 \mathrm{cc}$ & V24 Gy $<30 \%$ \\
V12 Gy $<20 \%$ & V12 Gy $<15 \%$ & Dmax $\left(35 \mathrm{~mm}^{3}\right)<39$ Gy \\
& & V36 Gy $<1 \mathrm{cc}$
\end{tabular}

volume will be obtained by an expansion of $2 \mathrm{~mm}$ around the CTV in this repeat radiotherapy context. This margin involves that the probability of coverage at 25,30 or $36 \mathrm{~Gy}$ is low, so the reporting will include D2\%, D50\%, D98\% and $\mathrm{D} 95 \%$ to describe as much as possible delivered dose. Organs at risk constraints are specified in table 1. Daily image-guided radiation therapy is mandatory, intra fraction tracking is recommended.

Quality control is particularly important in this setting of repeat radiotherapy. Before starting patient enrolments a 'dummy-run' will be conducted: an anonymous clinical chart will be forwarded to all participating sites with clinical, scintigraphy (choline-PET scan), CT scan and MRI data prior to repositioning. After delineating the relevant volumes, each site will have to perform a dosimetry which will be centralised in order to verify that the constraints are being observed. For each site, the dosimetric data will be subject to a centralised review prior to SBRT administration in order to verify that constraints are being observed. Follow-up visits are described in figures 1 and 2.

\section{Sample size calculation}

Required number of patients to be included: minimum 47 patients. The total sample size will depend on the number of patients allocated at the different dose levels in the dose-finding parts of the trial. A minimum of 13 patients will be recruited before the expansion phase is open (phase II part). A total of 44 patients allocated at the recommended dose (recruited in the dose-escalation stage or in the expansion phase) and evaluable at 3 years are required for the main analysis of the phase II part of the trial to ensure an $85 \%$ power if 3 -year bRFS is $\mathrm{p} 1=0.70$, with a test against $\mathrm{p} 0=0.50$ at a one-sided $5 \%$ alpha level.

\section{Statistical considerations}

Phase I

Three dose levels of SBRT are to be considered: $5 \times 6,6 \times 6$ and $5 \times 5 \mathrm{~Gy}$. The starting dose level is $5 \times 6 \mathrm{~Gy}$. The lower dose level, $5 \times 5 \mathrm{~Gy}$, will be considered if excessive toxicity is reported at the first dose level $(5 \times 6 \mathrm{~Gy})$. A TITE-CRM with an empiric dose-toxicity model in a Bayesian framework will be used for the dose-finding part of the trial to identify the recommended dose. The target DLT probability is set at $p(D L T)=0.25$. Observations of patients who have no DLT at the time of the analysis but have not completed the DLT assessment period will be down-weighted in the likelihood, proportionally to the length of follow-up; for instance, if the last patient has been recruited 8 weeks before a new patient is available for enrolment, and is evaluated at week 10 with no DLT, then his observation is attributed a weight of $10 / 18=0.56$. 


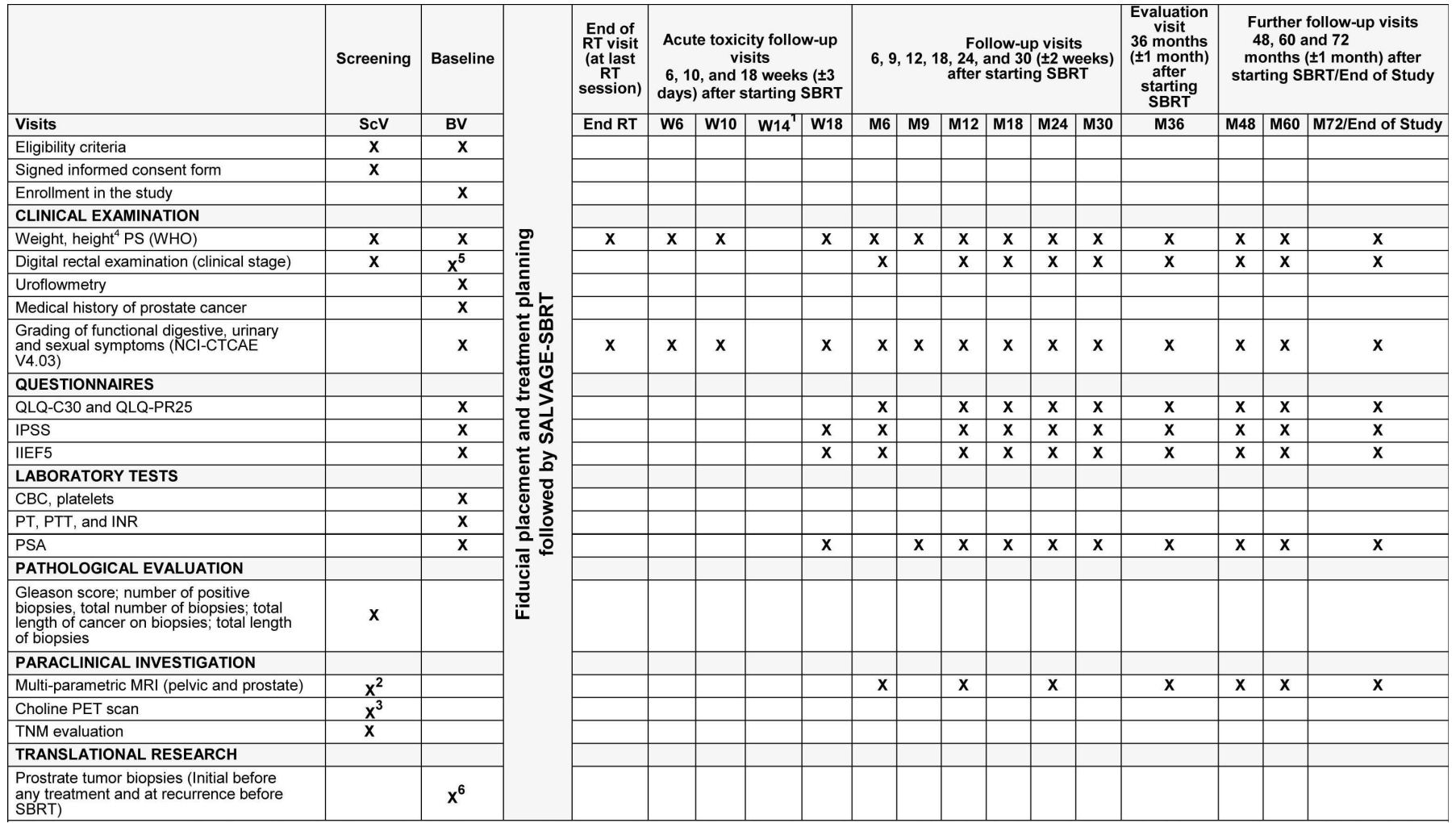

Figure 2 Detailed description of study flowchart. (1. Patient will be contacted by phone 14 weeks after starting SBRT to assess toxicity; an additional visit may be scheduled if required. 2. Before inclusion, during regular follow-up and in case of biochemical recurrence after SBRT. 3. Before inclusion and in case of biochemical recurrence after SBRT. 4. Patient's height will only be measured at the screening visit. 5 . If not done at the screening visit. 6 . Only applicable for patients who have consented to participate in the biological ancillary study). SBRT, stereotatic body radiotherapy.

At least three patients will be enrolled at the first doselevel and fully evaluated over the 18-week study period before the dose is escalated to the next dose level. Radiation dose levels for further patients will be defined based on the estimate of the probability of DLT at each dose level considering all available information accumulated so far. Patients will be treated at the best current estimate of the recommended dose, that is, the dose associated with an estimated probability of DLT the closest to the target 0.25 . Patients may eventually be treated at a dose below the dose recommended by the model for safety reasons. The patients can be recruited with no minimal time interval between successive inclusions. During the dose-escalation part of the trial, safety data will have to be reported in the data base in real time. A monthly teleconference meeting with the participation of the biostatistician, the trial coordinator and a representative of the sponsor, to summarise toxicity observations and define the dose to be allocated to the next patient(s) will be held. Safety data will be discussed with an Independent Data Monitoring Committee at the end of the dose-escalation part of trial, or before if needed.

The dose-escalation part of the study will terminate once 10 patients have been treated and evaluated at a dose currently identified as the recommended dose. Further patients will then be accrued in the expansion cohort, where toxicity data collected over the first 18-week period and DLT assessment will be analysed approximately every 10 patients with the possibility of modification of the recommended dose, based on model-based estimates.

Specifications of the model are detailed in online supplementary appendix, as well as the results of a simulation study evaluating the operating characteristics of the proposed design.

\section{Phase II}

The phase II is based on a one-stage design based on an efficacy endpoint (3-year bRFS). Assuming that information will be available for all patients at 3 years, the endpoint follows a binomial distribution. The design was thus defined considering exact tests, as published by A'Hern. ${ }^{39}$

From the literature, the expected bRFS rate at 3 years is $~ 50 \%$ in this patient population with various salvage therapies. A 3-year bRFS $\leq 0.50$ is deemed insufficient for further evaluation of this approach $(\mathrm{p} 0=0.50)$. The considered alternative hypothesis is $\mathrm{p} 1=0.70$.

The phase II part of the study will need to include 44 patients (including the patients recruited in the dosefinding part of the phase I, allocated at the dose level finally identified as the recommended dose). If all 44 patients are evaluable at 3 years, the treatment strategy will be considered to be insufficiently effective if $\leq 27$ patients are alive without a biochemical relapse at 3 years. 
The operating characteristics of the design are:

1. $\mathrm{p} 0=0.50, \mathrm{p} 1=0.70$.

2. Defined type I error $=0.05$ (computed type-I error of the design $=0.048$ ).

3. Defined power $=0.85$ (computed power $=0.861$ ).

If some patient data are censored before 3 years, the 3-year bRFS will be estimated using Kaplan-Meier method and the lower boundary of the $90 \%$ CI will be compared with $\mathrm{p} 0=0.50$. The conclusion will be positive if we can reject the null hypothesis $\mathrm{p} 0=0.50$ at a one-sided $5 \%$ alpha level.

\section{Patient and public involvement}

Patients were not involved in the idea conception of this trial.

Patients were not involved in the design of this study nor in recruitment of the study.

\section{ETHICS AND DISSEMINATION}

The study has been submitted and approved by ethics committee 'Ile de France III' (2017-A00008-45) for all study sites. A written informed consent will be obtained from the study participants. In France, according to the current law, a protocol can be subjected to any regional ethics committee, even if no hospital of this region takes part to the trial. The choice is made according to the workload of every committee. The opinion of this ethics committee applies to all the national centres. Academic dissemination will occur through publication and conference presentations.

\section{DISCUSSION}

To date, no standard local treatment exists for patients with an intraprostatic recurrence after radiotherapy. A number of treatments options exist including RP, brachytherapy, HIFU, cryotherapy and more recently SBRT. These treatments are associated with a variety of genitourinary and gastrointestinal toxicities and complications. The literature to date consists mainly of retrospective and small prospective series making it difficult to assess and compare these techniques. Philippou et al compared oncological and toxicity outcomes of SRP versus non-surgical therapies except SBRT with a meta-regression analysis. Oncological and toxicity outcomes appear to be similar; however, all non-surgical salvage modalities may be associated with better continence outcomes. ${ }^{40}$

The sensitivity of PSMA-PET is higher than that of choline-PET for the detection of lymph node disease. ${ }^{41}$ Unfortunately, PSMA-PET is not routinely available in France. A modification of the inclusion criteria is being drafted to allow PSMA-PET if this examination becomes available during the study period. To have a high sensitivity, a surgical lymph node staging must be extensive, which can have side effects in this context. Furthermore, clinical and biological selection criteria (no high-risk cancer before first treatment, PSA level, PSA doubling time and so on) are designed to select the patients to have most likely intraprostatic recurrence only.

The phase I part of GETUG-AFU 31 trial will evaluate SBRT dose of $30 \mathrm{~Gy}$ in 5 fractions, over 12 days, and then depending on tolerance, $36 \mathrm{~Gy}$ in 6 fractions over 12 days. The latter, 36 Gy in 6 fractions, is the current scheme used at the Oscar Lambret Centre for all repeat SBRT. This dose is higher than that described by Jereczek-Fossa $e t$ al (30 Gy in 5 fractions), ${ }^{29}$ discussed as being too low, ${ }^{30}$ but lower than the dose used by Fuller et al (38 Gy in 4 fractions).$^{30} \mathrm{~A}$ lower dose $(5 \times 5 \mathrm{~Gy})$ was used in Zerini $e t$ $a l .{ }^{32}$ We have decided to initially use a phase I study, using DLT criteria, to establish the best dose (either $5 \times 6,6 \times 6$ or $5 \times 5 \mathrm{~Gy}$ ) for the phase II part of the study. In phase I radiotherapy trials, late complications are often not taken into account and there is currently no consensus on the methodology used for these studies. Although most phase I radiotherapy studies use a $3+3$ design, it is not suitable for these studies as it does not assess late toxicity. More complex designs such as the TITE-CRM are recommended, which will shorten the duration of the entire trial and efficiently uses patient information throughout the study. ${ }^{42}$

\section{Author affiliations}

${ }^{1}$ Academic Department of Radiation Oncology, Centre Oscar Lambret, Lille, France

${ }^{2}$ CRIStAL UMR CNRS 9189, Lille University, Villeneuve-d'Ascq, France

${ }^{3}$ Methodology and Biostatistic Unit, Centre Oscar Lambret, Lille, France

${ }^{4}$ Department of Urology, Centre Hospitalier Universitaire de Dijon, Dijon, France

${ }^{5}$ CNRS UMR 8161, Institut de Biologie de Lille, Lille, France

${ }^{6}$ UNICANCER, Paris, France

Contributors Study conception: DP. Revision of study design and protocol: DP, MCLD, ET, LC, MD, SN and EL. Study coordination: DP, MCLD, ET and SN. All authors read and approved the final manuscript.

Funding This study is funded by a grant of National Institute of Cancer INCA (INCaDGOS_9816). The funding body had no role in the design of the study, collection, analysis, and interpretation of data and in writing the manuscript.

Competing interests None declared.

Patient consent for publication Obtained.

Provenance and peer review Not commissioned; externally peer reviewed.

Open access This is an open access article distributed in accordance with the Creative Commons Attribution Non Commercial (CC BY-NC 4.0) license, which permits others to distribute, remix, adapt, build upon this work non-commercially, and license their derivative works on different terms, provided the original work is properly cited, appropriate credit is given, any changes made indicated, and the use is non-commercial. See: http://creativecommons.org/licenses/by-nc/4.0/.

\section{REFERENCES}

1. Malvezzi M, Bertuccio P, Rosso T, et al. European cancer mortality predictions for the year 2015: does lung cancer have the highest death rate in Eu women? Ann Oncol 2015;26:779-86.

2. Zelefsky MJ, Reuter VE, Fuks Z, et al. Influence of local tumor control on distant metastases and cancer related mortality after external beam radiotherapy for prostate cancer. J Urol 2008;179:1368-73. discussion 1373.

3. Kass-Iliyya A, Jovic G, Murphy C, et al. Two-years postradiotherapy biopsies: lessons from MRC RT01 trial. Eur Urol 2018;73:968-76.

4. D'Amico AV, Cote K, Loffredo M, et al. Pretreatment predictors of time to cancer specific death after prostate specific antigen failure. $J$ Urol 2003;169:1320-4. 
5. Buyyounouski MK, Hanlon AL, Horwitz EM, et al. Interval to biochemical failure highly prognostic for distant metastasis and prostate cancer-specific mortality after radiotherapy. Int J Radiat Oncol Biol Phys 2008;70:59-66.

6. Roach M, Hanks G, Thames $\mathrm{H}$, et al. Defining biochemical failure following radiotherapy with or without hormonal therapy in men with clinically localized prostate cancer: recommendations of the RTOGASTRO Phoenix consensus conference. Int J Radiat Oncol Biol Phys 2006;65:965-74.

7. Chade DC, Eastham J, Graefen M, et al. Cancer control and functional outcomes of salvage radical prostatectomy for radiationrecurrent prostate cancer: a systematic review of the literature. Eur Urol 2012;61:961-71.

8. Gotto GT, Yunis LH, Vora K, et al. Impact of prior prostate radiation on complications after radical prostatectomy. J Urol 2010;184:136-42.

9. Yamada Y, Okihara K, Iwata T, et al. Salvage brachytherapy for locally recurrent prostate cancer after external beam radiotherapy. Asian $J$ Androl 2015;17:899-903.

10. Yamada $Y$, Kollmeier MA, Pei X, et al. A phase II study of salvage high-dose-rate brachytherapy for the treatment of locally recurrent prostate cancer after definitive external beam radiotherapy. Brachytherapy 2014:13:111-6.

11. Wojcieszek P, Szlag M, Głowacki G, et al. Salvage high-dose-rate brachytherapy for locally recurrent prostate cancer after primary radiotherapy failure. Radiother Oncol 2016;119:405-10.

12. Crook JM, Zhang P, Pisansky TM, et al. A prospective phase 2 trial of Transperineal ultrasound-guided brachytherapy for locally recurrent prostate cancer after external beam radiation therapy (NRG/ RTOG0526): initial report of late toxicity outcome. Int $J$ Radiat Oncol Biol Phys 2017;99:S1.

13. Brachytherapy for recurrent prostate cancer (CAPRICUR). Available: https://clinicaltrials.gov/ct2/show/NCT01956058 [Accessed 5 Feb 2018].

14. van Velthoven $\mathrm{R}$, Aoun $\mathrm{F}$, Marcelis $\mathrm{Q}$, et al. A prospective clinical trial of HIFU hemiablation for clinically localized prostate cancer. Prostate Cancer Prostatic Dis 2016;19:79-83.

15. Crouzet S, Murat F-J, Pommier P, et al. Locally recurrent prostate cancer after initial radiation therapy: early salvage high-intensity focused ultrasound improves oncologic outcomes. Radiother Oncol 2012;105:198-202.

16. Finley DS, Belldegrun AS. Salvage cryotherapy for radiation-recurrent prostate cancer: outcomes and complications. Curr Urol Rep 2011;12:209-15.

17. Pisters LL, Rewcastle JC, Donnelly BJ, et al. Salvage prostate cryoablation: initial results from the cryo on-line data registry. $J$ Urol 2008;180:559-64. discussion 563-4.

18. Pisters LL, Leibovici $\mathrm{D}$, Blute $\mathrm{M}$, et al. Locally recurrent prostate cancer after initial radiation therapy: a comparison of salvage radical prostatectomy versus cryotherapy. J Urol 2009;182:517-27. discussion 525-7.

19. Lian H, Yang R, Lin T, et al. Salvage cryotherapy with third-generation technology for locally recurrent prostate cancer after radiation therapy. Int Urol Nephrol 2016;48:1461-6.

20. Jaswal J, Crook J. The role of intermittent androgen deprivation therapy in the management of biochemically recurrent or metastatic prostate cancer. Curr Urol Rep 2015;16:11.

21. Agarwal PK, Sadetsky N, Konety BR, et al. Cancer of the prostate strategic urological research endeavor (CaPSURE). treatment failure after primary and salvage therapy for prostate cancer: likelihood, patterns of care, and outcomes. Cancer 2008:112:307-14.

22. Oefelein MG, Ricchiuti V, Conrad W, et al. Skeletal fractures negatively correlate with overall survival in men with prostate cancer. J Urol 2002;168:1005-7.

23. Berruti A, Dogliotti L, Tucci M, et al. Metabolic bone disease induced by prostate cancer: rationale for the use of bisphosphonates. $J$ Urol 2001;166:2023-31.
24. European Association of Urology. Prostate cancer guidelines. Available: http://uroweb.org/guideline/prostate-cancer/\#6_9 [Accessed 5 Feb 2018].

25. King CR, Freeman D, Kaplan I, et al. Stereotactic body radiotherapy for localized prostate cancer: pooled analysis from a multiinstitutional Consortium of prospective phase II trials. Radiother Oncol 2013;109:217-21.

26. Fuller DB, Naitoh J, Mardirossian G. Virtual HDR CyberKnife SBRT for localized prostatic carcinoma: 5-year disease-free survival and toxicity observations. Front Oncol 2014;4:321.

27. King CR, Collins S, Fuller D, et al. Health-Related quality of life after stereotactic body radiation therapy for localized prostate cancer: results from a multi-institutional Consortium of prospective trials. Int J Radiat Oncol Biol Phys 2013;87:939-45.

28. Katz AJ, Santoro M, Diblasio F, et al. Stereotactic body radiotherapy for localized prostate cancer: disease control and quality of life at 6 years. Radiat Oncol 2013;8.

29. Jereczek-Fossa BA, Beltramo G, Fariselli L, et al. Robotic imageguided stereotactic radiotherapy, for isolated recurrent primary, lymph node or metastatic prostate cancer. Int J Radiat Oncol Biol Phys 2012;82:889-97.

30. Fuller DB, Wurzer J, Shirazi R, et al. High-dose-rate stereotactic body radiation therapy for postradiation therapy locally recurrent prostatic carcinoma: preliminary prostate-specific antigen response, disease-free survival, and toxicity assessment. Pract Radiat Oncol 2015;5:e615-23.

31. Leroy T, Lacornerie T, Bogart E, et al. Salvage robotic SBRT for local prostate cancer recurrence after radiotherapy: preliminary results of the OSCAR Lambret center. Radiat Oncol 2017;12.

32. Zerini D, Jereczek-Fossa BA, Fodor C, et al. Salvage image-guided intensity modulated or stereotactic body reirradiation of local recurrence of prostate cancer. Br J Radiol 2015;88:20150197.

33. Punnen S, Cooperberg MR, D'Amico AV, et al. Management of biochemical recurrence after primary treatment of prostate cancer: a systematic review of the literature. Eur Urol 2013;64:905-15.

34. Smith M, Bernstein M, Bleyer WA, et al. Conduct of phase I trials in children with cancer. J Clin Oncol 1998;16:966-78.

35. Cheung YK, Chappell R. Sequential designs for phase I clinical trials with late-onset toxicities. Biometrics 2000;56:1177-82.

36. O'Quigley J, Pepe M, Fisher L. Continual reassessment method: a practical design for phase 1 clinical trials in cancer. Biometrics 1990;46:33-48.

37. Wachter S, Gerstner N, Goldner G, et al. Endoscopic scoring of late rectal mucosal damage after conformal radiotherapy for prostatic carcinoma. Radiother Oncol 2000;54:11-19.

38. Goldner G, Tomicek B, Becker G, et al. Proctitis after external-beam radiotherapy for prostate cancer classified by Vienna Rectoscopy score and correlated with EORTC/RTOG score for late rectal toxicity: results of a prospective multicenter study of 166 patients. Int $J$ Radiat Oncol Biol Phys 2007;67:78-83.

39. A'Hern RP. Sample size tables for exact single-stage phase II designs. Stat Med 2001;20:859-66.

40. Philippou Y, Parker RA, Volanis D, et al. Comparative oncologic and toxicity outcomes of salvage radical prostatectomy versus nonsurgical therapies for radiorecurrent prostate cancer: a metaregression analysis. Eur Urol Focus 2016;2:158-71.

41. Lecouvet FE, Oprea-Lager DE, Liu Y, et al. Use of modern imaging methods to facilitate trials of metastasis-directed therapy for oligometastatic disease in prostate cancer: a consensus recommendation from the EORTC imaging group. Lancet Oncol 2018;19:e534-45.

42. Pijls-Johannesma M, van Mastrigt G, Hahn SM, et al. A systematic methodology review of phase I radiation dose escalation trials. Radiother Oncol 2010;95:135-41. 